\title{
Carbon Dioxide Absorber Device
}

National Cancer Institute

\section{Source}

National Cancer Institute. Carbon Dioxide Absorber Device. NCI Thesaurus. Code C49804.

A device or material designed to sequester carbon dioxide. 